\title{
ATYPICALITY IN PRESENTATION OF NEUROLEPTIC MALIGNANT SYNDROME CAUSED BY OLANZAPINE
}

\author{
BISWARANJAN MISHRA, BAIKUNTHANATH MISHRA*, SADDICHHA SAHOO, MANU ARORA, \\ C. R. J. KHESS
}

\section{ABSTRACT}

N euroleptic malignant syndrome (NMS) is the most serious of acute neurological side effects produced by antipsychotic medication, characterized by hyperthermia, rigidity, altered consciousness and autonomic dysfunction, the prevalence of which varies from 0.4-1.4\%. NMS is usually seen in treatment with high potency typical antipsychotics and very rarely with atypical antipsychotics. However, NMS cases have been reported with risperidone, clozapine, olanzapine and quetiapine. The presentations of N MS have often varied, and we report another atypicality in presentation of NMS due to olanzapine use.

Key words: Amantadine, electroconvulsive therapy, neuroleptic malignant syndrome, olanzapine

\section{INTRODUCTION}

Neuroleptic malignant syndrome (NMS) is the most serious of acute neurological side effects produced by antipsychotic medications, characterized by hyperthermia, rigidity, altered consciousness and autonomic dysfunction, ${ }^{[1]}$ the prevalence of which varies from $0.4-1.4 \%{ }^{[2]}$ NMS is usually seen in treatment with high potency typical antipsychotics and very rarely with atypical antipsychotics. ${ }^{[3]}$ However, NMS cases have been reported with risperidone, ${ }^{[4]}$ clozapine, ${ }^{[4]}$ olanzapine ${ }^{[4,5]}$ and quetiapine. ${ }^{[6]}$

Dept. of Psychiatry, Central Institute of Psychiatry,

Ranchi; *Som Hospital, Bhubaneswar, India

\section{Correspondence:}

Dr. Sahoo Saddichha, Central Institute of Psychiatry, Ranchi, India. E-mail: saddichha@gmail.com
We report another atypicality in presentation of NMS due to olanzapine use.

\section{CASE REPORT}

A 21-year-old male, was diagnosed as mania with psychotic symptoms, drug naïve on admission, was initially treated for 3 days with injectable haloperidol $10 \mathrm{mg}$ intramuscular b.i.d. for the control of agitation and aggressive behavior. He had no past history of any psychiatric or medical illness. He was subsequently started on oral olanzapine 10 mg. However, on the seventh day of treatment with Olanzapine, he was found to have fever (temperature 98.4-99.6 ${ }^{\circ} \mathrm{F}$.), tachycardia (pulse rate: 100-120/min, regular), blood pressure fluctuations (150-110 SBP/ 80-100 
DBP $\mathrm{mmHg}$.), generalized rigidity, increased salivation and diaphoresis, along with normal orientation.

The patient was suspected to be having NMS. Other differential diagnoses such as malignant hyperthermia, lethal catatonia, heat stroke, tetanus, CNS infections, septicemia, etc., however, were clinically ruled out. He had no physical or muscular injury. Laboratory investigations revealed the following: TLC12600/cu. mm, Neutrophil-85\%, CPKMB-37 $\mu \mathrm{g} / \mathrm{L}(0-7 \mu \mathrm{g} / \mathrm{L})$, SGOT-119 IU/L (0-45 IU/L), SGPT-66 IU/L (0-35 IU/L) and ECG suggesting sinus tachycardia.

Olanzapine was stopped and adequate hydration was maintained. In view of the unavailability of dantroline, amantadine 100 mg b.i.d. was added. Within 3 days the patient showed improvement in terms of autonomic stability, absence of fever and reduction in rigidity. However, on the fifth day there was recurrence of the generalized rigidity. Hence bilateral electroconvulsive therapy (ECT) was started, with patient showing adequate improvement in all the symptoms of NMS after 3 ECTs, following which he was put on quetiapine, gradually hiked to $200 \mathrm{mg}$ / day; and sodium valproate $1000 \mathrm{mg}$. All investigations, including CPK, were repeated after 1 week and were found to be within normal limits. Patient showed significant improvement in his clinical state and is maintaining well after 4 weeks of discharge from the hospital.

\section{DISCUSSION}

NMS was considered in this patient in view of the clinical triad of fever, rigidity and autonomic instability, along with supportive laboratory findings of leukocytosis and raised CPK levels. Our patient also had all the risk factors described for NMS - namely, young age and being male ${ }^{[1]}$ and the presence of affective illness and agitation. ${ }^{[1]}$ Another differential diagnosis, lethal catatonia, was discarded since our patient was exposed to neuroleptics from the day of admission and which can only be considered if a person has NMS-like presentation in the absence of neuroleptic exposure. ${ }^{[7]}$ Further, lethal catatonia begins with extreme psychotic excitement, whereas neuroleptic malignant syndrome begins with severe muscle rigidity, ${ }^{[4]}$ as seen in our patient.

Although extrap seen in our pa

Although extrapyramidal symptoms such as tremors, salivation, diaphoresis and orthostatic hypotension can be commonly seen with the use of olanzapine, presence of additional features such as severe muscular rigidity and fever helped us reach a diagnosis of NMS. As NMS is a symptom spectrum with varying levels of severity, several diagnostic criteria have been proposed. ${ }^{[4]}$ The most agreed upon criteria are the combination of 'muscle rigidity and elevated temperature, which may range from mild elevations to markedly hyperthermic states $\left(99-106^{\circ} \mathrm{F}\right),{ }^{,[1]}$ along with two or more of other supportive findings such as orthostatic dysregulation, tremor, diaphoresis, leukocytosis and elevated CPK levels ${ }^{[1]}$ - all of which were seen in our patient.

However, a review of NMS cases with atypical antipsychotics ${ }^{[9]}$ reveals contradictory findings. The clinical presentation of NMS due to clozapine differs from that due to conventional antipsychotics in that there are fewer extrapyramidal symptoms. ${ }^{[10]}$ On the contrary, Hasan and Buckley ${ }^{[10]}$ suggest that NMS due to atypical antipsychotics resembles 'classical' NMS although there is higher CK elevation in the latter.

Although initial treatment with high potency antipsychotics such as haloperidol may cause NMS, we believe that the offending agent was Olanzapine, since the risk of developing NMS is the highest in the first week following antipsychotic exposure ${ }^{[1]}$ and our patient developed the symptoms more than a week after discontinuing haloperidol and during treatment with olanzapine. The earlier case report of olanzapine-induced $\mathrm{NMS}^{[5]}$ has also suggested an atypical form of NMS in the form of fluctuating hyperthermia, mild rigidity and no elevation of Creatinine Kinase. But our patient had marked rigidity and autonomic instability with absence of marked elevation of body temperature and Creatinine Kinase, an atypicality rarely observed.

Amantadine was considered for treatment of NMS since dopaminergic depletion has been postulated as the etiology in NMS and managed with the dose of $100 \mathrm{mg} 1$ 3 times a day. ${ }^{[8]}$ This initially showed good response in reducing the muscular rigidity, but subsequently there was a plateau. Since NMS is hypothesized as being due to dopaminergic blockade, ${ }^{[4]}$ the plateau in response of Amantadine may possibly be due to its weak dopaminomimetic effects, ${ }^{[11]}$ for which other treatment options were considered, such as ECT. The beneficial effect of ECT in NMS has been suggested due to an increase in receptor sensitivity to dopamine, ${ }^{[12]}$ with earlier reports of successful treatment of atypical antipsychoticsinduced NMS. ${ }^{[13]}$ Hence ECT was considered in our patient to bring about complete resolutio of NMS after partial response to Amantadine. Another point to favor ECT was the fact that it is also useful for rapid control of manic and psychotic agitation. ${ }^{[12]}$

NMS can be considered as a spectrum disorder in which the core features of rigidity, hyperthermia, autonomic instability and elevated Creatinine Kinase can present in varying degrees of severity. Olanzapine can produce NMS with atypical presentations. The concomitant use of ECT along with amantadine is effective fo complete resolution of symptoms of NMS.

\section{REFERENCES}

1. American Psychiatric Association: Diagnostic and Statistical Manual of Mental Disorders (DSM-IV TR), $4^{\text {th }}$ ed. American Psychiatric Association: Washington, DC; 2000.

2. Pope HG Jr, Keck PE Jr, McElroy SL. Frequency and presentation of neuroleptic malignan syndrome in a large psychiatric hospital. Am Psychiatry 1986;143:1227-33.

3. al-Waneen R. Neuroleptic malignant syndrom associated with quetiapine. Can J Psychiatry 2000;45:764-5.

4. Pelonero AL, Levenson JL, Pandurangi AK. Neuroleptic malignant syndrome: A review. Psychiatr Serv 1998;49:1163-72.

5. Nielsen J, Bruhn AM. Atypical neuroleptic malignant syndrome caused by olanzapine. Acta Psychiatr Scand 2005;112:238-40.

6. Sing KJ, Ramaekers GM, Van Harten PN. Neuroleptic malignant syndrome and quetiapine. Am J Psychiatry 2002;159:149-50.

7. White DA. Catatonia and the neuroleptic malignan syndrome: A single entity? $\mathrm{Br} J$ Psychiatry 1992;161:558-60.

8. Lazarus A, Mann SC, Caroff SN. The neuroleptic malignant syndrome and related conditions. 
American Psychiatric Press: Washington, DC; 1989.

9. Kontaxakis VP, Havaki-Kontaxaki BJ, Christodoulou NG, Paplos KG. Olanzapine-associated neuroleptic malignant syndrome. Prog Neuropsychopharmacol Biol Psychiatry 2002;26:897-902.

10. Hasan S, Buckley P. Novel antipsychotics and the neuroleptic malignant syndrome: A review and critique. Am J Psychiatry 1998;155:1113-6.

11. Weller M, Kornhuber J. A rationale for NMDA receptor antagonist therapy of the neuroleptic malignant syndrome. Med Hypotheses
1992;38:329-33.

12. Davis JM, Janicak PG, Sakkas P, Gilmore C, Wang Z. Electroconvulsive Therapy in the Treatment of the Neuroleptic Malignant Syndrome. Convul Ther 1991;7:111-20.

13. Bora E, Saffet A, Fisun G. Neuroleptic malignantlike syndrome induced with low-dose quetiapine treated with electroconvulsive therapy. Eur Psychiatry 2003;18:323.

Source of Support: Nil, Conflict of Interest: None declared. 